\title{
Motivadores de consumo de alcohol en adolescentes: análisis de diferencias inter-género y propuesta de un continuum etiológico
}

\author{
María de la Villa Moral Jiménez*; francisco Javier Rodríguez Díaz**; Carlos Sirvent Ruiz*** \\ Universidad de Oviedo e Instituto Spiral \\ * Profesora Asociada Doctora. Área de Psicología Social. Universidad de Oviedo. \\ * Profesor Titular. Área de Personalidad, Evaluación y Tratamiento. Universidad de Oviedo. \\ ** Psiquiatra. Director de la Fundación Instituto de Drogodependencias Spiral (Oviedo y "Centro Cibeles", Madrid)
}

Enviar correspondencia a:

María de la Villa Moral Jiménez. Universidad de Oviedo. Facultad de Psicología. Plaza Feijóo, s/n. despacho 211. 33003 OVIEDO. Tel. (98) 5103282. Fax (98) 5104144 - (98) 5104141. E-mail: psico4@correo.uniovi.es - mvilla@uniovi.es

Recibido: 9 de febrero de 2004 Aceptado: 4 de octubre de 2004

\section{RESUMEN}

Se analizan los principales motivadores de consumo de alcohol, desde una aproximación psicosociológica y clínica, y con la propuesta de un continuum etiológico de una problemática abordada comprehensivamente. Son exploradas las diferencias inter-género en la experimentación con alcohol y otras sustancias psicoactivas, así como en los motivadores de consumo. La muestra está compuesta por 273 estudiantes con edades que oscilan entre los catorce y los dieciocho años seleccionados de cuatro Centros de Secundaria del Principado de Asturias. De acuerdo con los resultados obtenidos, al explicitar las razones que inducen a la experimentación con alcohol, se priorizan variables asociadas al estado emocional, a la búsqueda de nuevas sensaciones y a la vinculación grupal. Las principales razones esgrimidas para no beber se concentran en intentos de evitación de problemas de salud física y conflictos familiares. En el análisis de las diferencias inter-género no se han obtenido diferencias significativas en los motivadores de consumo, salvo en su empleo para olvidar, y respecto a los hábitos de consumo las adolescentes fuman más, no hallándose diferencias significativas en un indicador como el número de borracheras experimentadas en el último mes o en el consumo de otras sustancias psicoactivas.

Palabras-clave: Alcohol, etiología, adolescencia, género, prevención.

\begin{abstract}
We analyse the principal relevant motivators for alcohol consumption, using a psychosociological and clinical approach, and with a proposal for an aetiological continuum to a comprehensively approached problematic. Gender differences in experimentation with alcohol and other psychoactive substances are explored, in addition to motivators for alcohol use. The sample comprises 273 students between fourteen and eighteen years of age, chosen from four secondary schools in Asturias. According to the results we obtained, when specifying the reasons leading to experimentation with alcohol, the primary variables were associated with the emotional state, the search for new sensations and to the influence of the group. The most important reasons given for not drinking were the prevention of potential health problems and family conflicts. Analysing gender differences, we find no significant differences in use motivators, with the exception of its use to forget. As for use habits, adolescents smoke more, there being no significant difference in such indicators as the number of episodes of drunkenness in the preceding month or in the use of other psychoactive substances.
\end{abstract}

Keywords: Alcohol, aetiology, adolescence, gender, prevention.

"Aparenta la virtud, si no la tienes; ... Refrénate esta noche; eso hará algo más fácil la próxima abstinencia, y aún más fácil la siguiente" W. Shakespeare. Hamlet.

\section{INTRODUCCIÓN}

E consumo de drogas institucionalizadas y de otras sustancias psicoactivas entre los adolescentes contemporáneos se vincula en la litera- tura sobre el tema a motivaciones hedónicas, intentos de satisfacción de búsquedas propias e inducidas, afanes explorativos, procesos de redefinición identitaria y necesidades de integración grupal en prácticas de ocio ritualizadas (Carrasco, 2000; Funes, 2000; Graña 
y Muñoz, 2000; Laespada, 2000, 2003; Martínez y Robles, 2001; Martínez, Fuertes, Ramos y Hernández, 2003; Moral, 2002; Moral y Ovejero, 2004; Sánchez, 2002). En las propuestas etiológicas sobre el consumo juvenil de sustancias psicoactivas se incurre con frecuencia en justificaciones interesadas del estatuto diferencial de las sustancias psicoactivas en función de su adscripción a la categoría de legal e ilegal, ya que se nos insta a ofrecer explicaciones mediante las que se fortalezcan representaciones sociales y discursivas dominantes. En cambio, ha de incidirse más bien en la nocividad de drogas legales como el alcohol o el tabaco de uso más extendido entre los adolescentes, cuya percepción de riesgo está distorsionada por mentalidades del usuario y conciencias permisivas, como se evidencia en los análisis de Bach i Bach (2000), Castellana y Lladó (1999), Pascual (2002) o Moral, Rodríguez y Sirvent (2004), entre otros.

Entre los principales factores determinantes del consumo juvenil de sustancias psicoactivas se le otorga especial significación a variables asociadas a la esfera actitudinal, al estado emocional y a la vinculación psicosocial del adolescente, así como a renovadas búsquedas y sobreactivaciones del tiempo de ocio juvenil (Espada, Méndez, Griffin y Botvin, 2003; García y Carrasco, 2002). Esta tendencia se justifica debido a la motivación netamente recreativa de ciertas experimentaciones con alcohol y otras sustancias psicoactivas asociadas a ciertas prácticas de esparcimiento juvenil de fin de semana (véase Bellis y Hughes, 2003; Calafat, Juan, Becoña, Fernández, Gil, Palmer et al., 2000; Calafat y Juan, 2003; Infante, Barro y Martín, 2003).

Desde el planteamiento psicosociológico que dirige esta aproximación se propone un continuum etiológico como acción retroalimentadora, donde prevalezcan los análisis comprehensivos sobre otros monocausales con pretensiones globalizantes. Individuo, contexto y sustancia no se deben desvincular en una propuesta etiológica de una problemática como ésta, ya que su interrelación se convierte en un criterio determinante básico. Se hace hincapié en la apelación al principio de la symploké, como acción de todo contenido en todo, donde los elementos implicados se van reformulando recursivamente y se redefinen en el otro del cual son parte constitutiva (Moral, 2002). Es decir, se aboga por no considerar la implicación aislada de variables orécticas, de personalidad o contextuales, sino por reconceptualizarlos como un producto multideterminado e imbricado. Tal interrelación de factores abarca un amplio espectro que incluye lo intraindividual, la vulnerabilidad genética y predisposiciones individuales asociadas a factores motivacionales, así como factores microsociales (refuerzo social, redes sociales, modelos negativos del grupo de iguales o familia, presión social, etc.) y a ellos se suman otros considerados individuales, tales como ciertos rasgos de per- sonalidad. Asimismo, como factores determinantes de carácter contextual actúa el modelado sociocultural y estados de inercia social relacionados con la acción de los mecanismos de control social, políticos o fácticos. Todo ello ha de ir incardinado en una propuesta vinculada a la concepción social de las acciones de salud, tal y como sugirió León Rubio (2000). En este tipo de propuestas comprehensivas no han de desdeñarse ni, por un lado, análisis de bases neurológicas de las drogodependencias (Fernández-Espejo, 2002) $u$ otros estudios relativos a la vulnerabilidad personal ante la drogadicción (Ambrosio, 2003), ni referencias a influencias macrosituacionales (disponibilidad, persuasión publicitaria, cultura de la droga, etc.) (Rooney, 1990; Sánchez-Carbonell, 2004) y macrotendencias asociadas a transformaciones tecnológicas, políticas o culturales que redefinen las coordenadas de la sociedad contemporánea (Berenzon, Medina-Mora, Carreño, Juárez, Rojas y Villatoro, 1996; Freixa, 2000). La búsqueda de factores etiológicos ha de concentrarse, simultáneamente, en un complejo entramado multifactorial de influencias, en un estado de pulsiones y motivaciones, junto a acciones externas intrusivas representadas en la acción de factores intra e interindividuales, sociales, históricos y culturales que son tales por el proceso dialéctico que los reconstituye.

Uno de los objetivos básicos de esta investigación es abordar la cuestión relativa a las diferencias inter-género en materia de experimentación con sustancias psicoativas, de ahí que haya de convenirse en que abundan las perspectivas de análisis transdisciplinares de la construcción intersubjetiva de las diferencias inter-género vinculadas a la cultura de la droga (Moral, Ovejero y Rodríguez, 2002), así como del propio universo femenino y sus simbolismos, visiones estereotípicas, sistemas ideológicos y representaciones que fortalecen las propias construcciones de género (véase De Crescenzo, 2002; Fischer, 2000; Juliano, 2001; Morris, 2000). Concretamente, en la experimentación con drogas entre el colectivo juvenil abundan las aproximaciones a las diferencias inter-género en los patrones de consumo de alcohol y otras sustancias psicoactivas y en la esfera actitudinal, ya sea vinculando el uso de drogas a la asertividad como habilidad interpersonal básica (Suelves y Sánchez-Turet, 2001), ofreciendo análisis acerca de la incidencia de factores personales y situacionales (Vega y Garrido, 2000), focalizando la atención en un análisis diferencial según el nivel de consumo de alcohol (Bríñez-Horta, 2001) o mediante la adopción de una perspectiva de análisis crítica con respecto al mistificado binomio joven-droga (Moral, 2002; Moral y Ovejero, 2004; Moral et al., 2002).

Últimamente, se ha observado un incremento de los consumos de drogas institucionalizadas como alcohol y tabaco entre los adolescentes y parece confirmarse que los varones son más precoces y reali- 
zan un mayor consumo habitual, cuestión asociada al mayor riesgo de intoxicación, abuso y dependencia alcohólica confirmada en la investigación de BríñezHorta (2001). Asimismo, recordemos que, de acuerdo con las tendencias de los últimos años, a lo anterior se suman altas tasas de prevalencia de consumo de cannabis (Bobes, Bascarán, González y Sáiz, 2000; Calafat, Juan, Becoña, Fernández, Gil y Llopis, 2000; Laespada, 2003; Elzo, Comas, Laespada, Salazar y Vielva, 2000) y una extensión del uso de psicofármacos y drogas de síntesis entre la población juvenil (Barrio, Bravo y de la Fuente, 2000; D.G.P.N.S.D., 2000, 2001, 2002; Fernández, Álvarez, Secades, Jiménez, Cañada, Donate et al., 2003; Gamella y Álvarez, 1999; García-Portilla, Sáiz, Paredes, Martínez y Bobes, 2003; Sáiz, González, Paredes, Delgado, López, Martínez et al., 2001; Sáiz, García-Portilla, Paredes y Bobes, 2003). En la literatura sobre el tema se conviene en que se han reducido sensiblemente las diferencias inter-género, de modo que incluso se suele asistir a una igualación, e incluso inversión, de los patrones de consumo en drogas institucionalizadas, tales como alcohol (DGPNSD, 2001, 2002; Laespada, 2000, 2003; Laespada y Salazar, 1999; Moral, 2002) y una superación de los niveles de consumo en otras como el tabaco (Moral, 2002; Suelves y Sánchez-Turet, 2001), hecho evidenciado en informes en los que se ha hallado una experimentación equiparable en sustancias como el cannabis (DGPNSD, 2001). En todo caso, hay que partir del reconocimiento de que las diferencias inter-género están moduladas por la cultura, la historia y la propia sociedad que (re)interpreta de forma diferencial discursos y modos de actuación e incurre en normalizaciones que se aceptan como cuasinaturales o mistifica otras prácticas demonizándolas.

En definitiva, en esta investigación se ha procedido a ofrecer un aproximación psicosociológica a la etiología del consumo de alcohol y otras sustancias psicoactivas por parte de un colectivo de adolescentes que atraviesan un período vital conflictuado por búsquedas y/o crisis de identidad, así como por la puesta en práctica de diversas manifestaciones de esparcimiento juvenil y vinculación grupal, y se ha investigado sobre los procesos de construcción de las diferencias intergénero en los motivadores asociados a la experimentación.

\section{MÉTODOS}

\section{Muestra}

En esta investigación se han seleccionado cuatro Centros de Secundaria del Principado de Asturias mediante un muestro intencional, participando en la experiencia de todo el colectivo de alumnos una totalidad de 273 adolescentes de 3 er curso de E.S.O. $(12.5 \%)$, de $4^{\circ}(10.6 \%)$. y de $1^{\circ}$ de Bachillerato que representan el $76.9 \%$ de la muestra y pertenecen a varios grupos escolares de diferentes niveles académicos con edades que oscilan entre 14 y 18 años, situándose la media en la edad de 16.30 años. De acuerdo con la adscripción en función del género ha de concretarse que 133 son varones que representan el $48.7 \%$ y 140 chicas que integran el $51.3 \%$ restante. La elección de tal colectivo de alumnos ha de justificarse por la intención de analizar los hábitos de consumo de alcohol y otras sustancias psicoactivas entre jóvenes que estuviesen atravesando el estadío de la adolescencia media.

\section{Variables Investigadas e Instrumentos de Evalua- ción}

Se ha administrado un cuestionario construido ad hoc por los investigadores que bajo la denominación "Escala de Actitudes y Hábitos de Consumo de alcohol" incluye análisis de diversos elementos interrelacionados en la propuesta etiológica. Se ha elaborado a partir de la revisión de otras escalas, principalmente del "Cuestionario de Actitudes hacia el consumo de sustancias psicoactivas" (Macià, Olivares y Méndez, 1993), adaptado de Macià (1986) ("Cuestionario de Actitudes acerca del alcohol y Cuestionario de Información"), en formato tipo Likert, siendo en nuestra escala el valor de Alpha para 251 casos válidos y 142 ítems de .9171. Dado nuestro interés investigador por una aproximación al análisis de las motivaciones que impulsan al consumo y de otros hábitos de consumo de alcohol, así como de las razones explicitadas para beber y de aquellas que pueden mediatizar semejantes usos, se ha procedido a investigar variables tales como aquéllas vinculadas a las actividades de ocio juvenil, a la percepción del grado de problematicidad derivado del consumo de diversas sustancias psicoactivas, un exhaustivo análisis de las actitudes ante el consumo, el conocimiento expresado en relación con las consecuencias negativas derivadas del consumo de alcohol y acerca de la información sobre las drogas (principales vías de acceso a la información), y un estudio de los principales motivadores tanto del primer consumo de alcohol de los adolescentes como de los sucesivos, así como de las motivaciones percibidas para beber o no beber, cuestiones éstas últimas en las que hemos focalizado el interés divulgativo de los resultados en este artículo.

\section{Procedimiento}

Durante un intervalo temporal de dos semanas, en horario convenido con los Departamentos de Orientación de los Centros y de acuerdo con los tutores, los 
investigadores entrenados al efecto procedieron a la administración de la "Escala de Actitudes y Hábitos de Consumo de alcohol" al colectivo de estudiantes especificado mediante la asignación de un código numérico, atendiendo a una necesidad de carácter funcional como es la implementación de un conjunto preventivo-didáctico, dado el carácter longitudinal de la investigación

\section{RESULTADOS}

En esta aproximación al fenómeno objeto de estudio, de acuerdo con los resultados obtenidos al proceder a explicitar las razones que inducen a la experimentación con alcohol y otras sustancias psicoactivas, se privilegia una motivación netamente psicosocial. Esta tendencia se confirma a partir de la revisión de los datos expuestos en la Tabla 1, ya que se otorga una gran importancia como motivador de consumo en la primera experimentación a la vinculación grupal y al efecto de contagio de los iguales, ya sea bajo modalidades de modelado (porque bebían mis amigos) o mediante mecanismos de acción más persuasivos e impositivos (porque me incitaron a ello).
Asimismo, entre las motivaciones que impulsaron el primer consumo de alcohol se privilegia la búsqueda de sensaciones y la curiosidad como variable mediatizadora (tan sólo un $28.9 \%$ de los adolescentes rechazan este tipo de motivador). La búsqueda de evasión de la rutina junto a la euforia y desinhibición o las ilusiones de fortaleza y vigor físico representan otras de las posibilidades indicadas, obteniéndose menores porcentajes de adhesión (porcentajes de rechazo en el nivel de respuesta nunca de 76.6\%, 78.4\%, 71.8\%, $89.0 \%$, respectivamente) a tales motivadores inductores del primer consumo. Resultados similares se obtienen al analizar la frecuencia de empleo de los motivadores de consumo citados en los consumos sucesivos privilegiándose el modelado de sus amigos $(88.3 \%)$ y su incitación al consumo (81.3\%), así como la búsqueda de sensaciones (81.7\%) y la propia curiosidad ante la experimentación (82.4\%) (porcentajes de respuesta obtenidos al considerar conjuntamente los niveles de respuesta pocas, bastante y muchas veces).

Las principales razones que impulsan a los adolescentes a beber o a mantener conductas de no-consumo son de muy diversa índole. Con objeto de indagar sobre esta cuestión, se analiza el grado de acuerdo mostrado con los principales motivos explicitados para beber y no beber cuyos resultados se exponen

Tabla 1. Frecuencia de empleo de los principales motivadores de consumo de alcohol en la primera experimentación y en sucesivas.

\begin{tabular}{|lccccc|}
\hline $\begin{array}{l}\text { MOTIVADORES DE CONSUMO } \\
\text { DE ALCOHOL }\end{array}$ & $\begin{array}{c}\text { Nunca } \\
\%\end{array}$ & $\begin{array}{c}\text { Pocas veces } \\
\%\end{array}$ & $\begin{array}{c}\text { Bastante } \\
\%\end{array}$ & $\begin{array}{c}\text { Muchas veces } \\
\%\end{array}$ & $\begin{array}{c}\text { Casi siempre } \\
\%\end{array}$ \\
\hline PRIMER CONSUMO & & & & & \\
Olvidar & 83.9 & 9.5 & 5.1 & 0.0 & 1.5 \\
Evadir la rutina & 76.6 & 10.6 & 6.2 & 1.5 & 5.1 \\
Porque bebían mis amigos & 53.8 & 23.1 & 14.3 & 5.5 & 3.3 \\
Porque me incitaron a ello & 70.0 & 15.0 & 7.7 & 2.9 & 4.4 \\
Para desinhibirme & 78.4 & 11.4 & 6.2 & 2.2 & 1.8 \\
Para ligar & 86.8 & 8.8 & 3.3 & 1.1 & 0.0 \\
Para sentir euforia fuerte & 71.8 & 12.5 & 11.0 & 2.9 & 1.8 \\
Para sentirme más fuerte & 89.0 & 7.0 & 2.2 & 1.1 & 0.7 \\
Por curiosidad & 28.9 & 22.7 & 18.3 & 8.8 & 21.2 \\
\hline CONSUMOS sucESIVOS & & & & & \\
Olvidar & 22.7 & 22.7 & 27.5 & 10.3 & 16.8 \\
Evadir la rutina & 29.7 & 25.6 & 23.4 & 13.9 & 7.3 \\
Porque beben sus amigos & 11.7 & 11.7 & 25.3 & 22.7 & 28.6 \\
Porque les incitaron a ello & 18.7 & 25.6 & 23.8 & 17.9 & 13.9 \\
Para desinhibirse & 26.7 & 19.8 & 23.8 & 14.7 & 15.0 \\
Para ligar & 24.5 & 19.4 & 27.8 & 15.0 & 13.2 \\
Para sentir euforia & 18.3 & 18.3 & 23.4 & 22.0 & 17.9 \\
Para sentirse más fuerte & 25.3 & 21.2 & 19.0 & 17.2 & 17.2 \\
Por curiosidad & 17.6 & 26.7 & 19.0 & 14.3 & 22.3 \\
& & & & & \\
\hline
\end{tabular}


en la Tabla 2. Una de las principales razones moduladora de semejantes usos es la propia vinculación grupal (25.0\%), así como el intento de utilizar la experimentación como medio para evadir la rutina (20.9\%) o sentir euforia (21.9\%) e incluso para olvidar $(22.0 \%)$ (niveles de respuesta conjuntos MA y A). En cambio, entre los grados de adhesión a posibles razones esgrimidas para no beber los adolescentes encuestados se atienen principalmente a razones de salud como factores inhibidores de posibles usos/abusos, de ahí que un $86.0 \%$ de los adolescentes muestra su grado de acuerdo (niveles de respuesta MA y A) con la afirmación de que no beberían porque es malo para la salud, resultado que en principio redunda en el reconocimiento de la sensibilización de los jóvenes ante el daño derivado del consumo, aunque puede que no refleje auténticas tomas de conciencia. Asimismo, los adolescentes encuestados son conscientes de los daños anejos a la instauración de una posible dependencia (porque es difícil dejarlo una vez que se ha empezado, 51.1\% de acuerdo, niveles de respuesta MA y A), lo cual se asocia el reconocimiento de que el alcohol provoca accidentes graves $141.9 \%$, MA; $12.3 \%, A)$, de ahí que sea una de las razones de los que están concienciados que influye en su conducta de consumo. En el otro extremo del continuo la implicación de condicionantes macrocontextuales se hace evidente al aludir al porcentaje de adhesión a un indicador como es el la propia disponibilidad de la sustancia mermada por cuestiones económicas, ya que un $37.9 \%$ de los adolescentes determinan que una de las razones para no beber es precisamente el encarecimiento de la bebida. Otras de las razones esgrimidas de índole psicosocial más significativas para no beber es la relativa a la evitación de las consecuencias sociofamiliares derivadas del consumo, así se ha constatado que un $75.0 \%$ de los adolescentes muestra su acuerdo (niveles de respuesta MA y A) con la afirmación de que evitan beber porque provoca problemas en la vida familiar y un 55.6\% (MA y A) reconoce que no consumen alcohol porque les molesta a sus padres, y es que, ciertamente, la implicación socializadora paterna en materia de prevención educativa del consumo se hace notoria.

A partir del análisis de las diferencias inter-género en cuestiones relacionadas con los motivadores para el consumo u otras razones asociadas al rechazo del mismo y principales hábitos de experimentación de alcohol y otras sustancias psicoactivas, se constata la existencia de diferencias significativas en las razones autopercibidas para beber exclusivamente en el indicador relativo a olvidar $(p=.0023)$, en el sentido de que las adolescentes reconocen consumir alcohol en algunas oportunidades como mecanismo de evitación del

Tabla 2. Análisis porcentual del grado de acuerdo con los motivadores de consumo de alcohol y las principales razones para no beber.

\begin{tabular}{|lrrrrr|}
\hline RAZONES EXPLICITADAS PARA BEBERY NO BEBER & MD \% & D \% & INS \% & A \% & MA \% \\
\hline MOTIVADORES DE CONSUMO & & & & & \\
Olvidar & 51.5 & 26.1 & 6.2 & 14.0 & 2.2 \\
Evadir la rutina & 41.4 & 27.5 & 10.3 & 19.4 & 1.5 \\
Porque beben mis amigos & 34.2 & 24.3 & 16.5 & 19.9 & 5.1 \\
Para desinhibirme & 41.0 & 27.8 & 15.8 & 12.8 & 2.6 \\
Para ligar & 48.2 & 31.3 & 11.4 & 7.4 & 1.8 \\
Para sentir euforia & 39.6 & 24.9 & 13.6 & 17.9 & 4.0 \\
Para sentirme más fuerte & 51.1 & 30.1 & 9.2 & 7.4 & 2.2 \\
\hline RAZONES PARA NO-BEBER & & & & & \\
Porque es malo para la salud & 2.9 & 8.1 & 2.9 & 47.4 & 38.6 \\
Porque es demasiado caro & 10.7 & 30.9 & 19.5 & 29.0 & 9.9 \\
Porque es difícil dejarlo una vez que se ha empezado & 8.8 & 22.0 & 17.9 & 34.8 & 16.5 \\
Porque a mis padres les molesta mucho que beba & 8.1 & 15.0 & 21.2 & 36.6 & 19.0 \\
Tomar alcohol engorda & 15.1 & 26.8 & 26.1 & 20.6 & 11.4 \\
Porque no me gusta su sabor & 18.0 & 35.7 & 16.9 & 17.3 & 12.1 \\
Porque sus efectos son molestos (resaca, vómitos, mareos..) & 6.6 & 14.7 & 12.5 & 40.7 & 25.6 \\
Porque beber alcohol provoca daño & 11.8 & 24.6 & 20.6 & 31.3 & 11.8 \\
Porque va contra mis creencias religiosas & 54.6 & 27.5 & 13.2 & 2.6 & 2.2 \\
Provoca accidentes graves & 4.8 & 7.0 & 4.0 & 42.3 & 41.9 \\
Porque provoca problemas en la vida familiar & 4.8 & 8.1 & 12.1 & 40.1 & 34.9 \\
\hline MD= Muy en Desacuerdo, D= Desacuerdo, INS= Inseguro, A= De Acuerdo, MA $=$ Muy de Acuerdo. & \multicolumn{3}{c}{} \\
\hline
\end{tabular}


displacer provocado en la esfera emocional. En cambio, de acuerdo con los resultados hallados en esta investigación, no se han encontrado diferencias significativas con respecto a los motivadores que impulsan a los chicos y las chicas adolescentes al consumo de alcohol, ni en experimentación de nuevas sensaciones, en evasión lúdica ni de la rutina, esparcimiento, efecto de modelado grupal, etc. Convendría puntualizar que en respuesta a la pregunta planteada ¿Por qué crees que bebe la gente? las diferencias inter-género halladas se concentran en las respuestas relativas de nuevo a olvidar ( $\mathrm{p}=.0002)$, en la dirección ya apuntada, así como mecanismo de desinhibición $(p=.0052)$ y en cuestiones relativas al modelado grupal $(p=.0001)$ y a otras conminaciones al consumo del grupo de iguales ( $p=.0049$ ). Estando las expectativas individuales moduladas por tendencias de grupo y colectivas, se conviene en que las drogas cumplen ciertas funcio- nes de integración grupal, al mismo tiempo que desvían de las normas explícitas que regulan sancionando los usos/abusos, básicamente, de sustancias no institucionalizadas. Asimismo, se han obtenido diferencias inter-género significativas al mostrar el grado de acuerdo con las principales razones esgrimidas para no beber, tal y como se recoge en la Tabla 3. En virtud de semejante tendencia se apunta que, mientras que los chicos se decantan por aludir a una cuestión de índole económica como es el excesivo precio de las bebidas, las adolescentes redundan en considerar que son cuestiones vinculadas a la instauración de una posible dependencia y otras asociadas a las consecuencias derivadas del consumo, ya sea sobre la salud física (accidentes, calorías, daño en general) como sobre el bienestar psicosocial (problemas en la convivencia familiar).

Tabla 3. Análisis de las diferencias inter-género en los motivadores de consumo de alcohol y en las principales razones para no beber.

\begin{tabular}{|lrlrl|}
\hline DIFERENCIAS INTER-GÉNERO & F & F prob. & VARÓN Media & MUJER Media \\
\hline RAZONES PARA BEBER & & & & \\
Olvidar & 9.490 & $.002^{*}$ & 1.68 & 2.101 \\
Evadir la rutina & 2.694 & .102 & 2.00 & 2.236 \\
Porque beben mis amigos & .879 & .350 & 2.30 & 2.446 \\
Para desinhibirme & .666 & .415 & 2.02 & 2.136 \\
Para ligar & 1.732 & .186 & 1.92 & 1.755 \\
Para sentir euforia & .046 & .830 & 2.20 & 2.236 \\
Para sentirme más fuerte & 1.289 & .257 & 1.72 & 1.863 \\
\hline MOTIVACIÓN PERCIBIDA PARA EL CONSUMO DE ALCOHOL & & & & \\
Por qué crees que bebe la gente? & & & & \\
Olvidar & 14.484 & $.000^{*}$ & 2.44 & 3.06 \\
Evadir la rutina & 1.130 & .289 & 2.35 & 2.51 \\
Porque beben sus amigos & 15.106 & $.000^{*}$ & 3.13 & 3.74 \\
Porque les incitaron a ello & 4.222 & $.040^{* *}$ & 2.66 & 2.99 \\
Para desinhibirse & 7.936 & $.005^{*}$ & 2.47 & 2.94 \\
Para ligar & 2.968 & $.086^{* * *}$ & 2.59 & 2.86 \\
Para sentir euforia & 2.262 & .134 & 2.90 & 3.15 \\
Para sentirse más fuerte & .274 & .601 & 2.75 & 2.84 \\
Por curiosidad & 1.894 & .170 & 2.85 & 3.06 \\
\hline RAZONES PARA NO BEBER & & & & \\
Porque es malo para la salud & 8.147 & $.004^{*}$ & 3.93 & 4.27 \\
Porque es demasiado caro & 12.730 & $.000^{*}$ & 3.27 & 2.72 \\
Porque es difícil dejarlo una vez que se ha empezado & 6.470 & $.011^{* *}$ & 3.09 & 3.46 \\
Porque a mis padres les molesta mucho que beba & .091 & .763 & 3.41 & 3.46 \\
Tomar alcohol engorda & 6.118 & $.014^{* *}$ & 2.68 & 3.04 \\
Porque no me gusta su sabor & .032 & .858 & 2.68 & 2.71 \\
Porque sus efectos son molestos (resaca, vómitos, mareos..) & 1.294 & .256 & 3.56 & 3.72 \\
Beber alcohol provoca daño & 3.497 & $.063^{* * *}$ & 2.92 & 3.20 \\
Va contra mis creencias religiosas & .205 & .651 & 1.68 & 1.73 \\
Provoca accidentes graves & 3.145 & $.077^{* * *}$ & 3.98 & 4.21 \\
Provoca problemas en la vida familiar & 3.398 & $.066^{* * *}$ & 3.80 & 4.04 \\
\hline * p<.01 ** p<.05*** p<.10 & & & & \\
\hline
\end{tabular}


Tabla 4. Análisis de las diferencias inter-género en la experimentación con alcohol y otras sustancias psicoactivas (g.I.=272).

\begin{tabular}{|lcccc|}
\hline DIFERENCIAS INTER-GÉNERO & F. & F prob. & VARÓN Media & MUJER Media \\
\hline HÁBITOS DE CONSUMO & & & & \\
Borracheras en el último mes & 3.425 & $.065^{* *}$ & 2.48 & 1.57 \\
Consumo de tabaco & 3.492 & $.063^{* *}$ & 4.62 & 4.97 \\
\hline CONSUMO DE OTRAS DROGAS & & & & \\
JUNTO CON ALCOHOL & & & & .189 \\
Tabaco & 6.947 & $.009^{*}$ & .144 & .329 \\
Cannabis & 1.227 & .269 & .023 & .007 \\
Drogas de síntesis & 1.135 & .288 & .015 & .007 \\
Cocaína & .397 & .529 & .023 & .014 \\
Otras sustancias psicoactivas & .267 & .606 & & \\
\hline$* p<.01 * * p<.05 * * *$ p $<.10$ & & & & \\
\hline
\end{tabular}

En la experimentación con alcohol y otras sustancias psicoactivas, de acuerdo con los resultados expuestos en la Tabla 4, se ha hallado que los chicos reconocen haberse emborrachado en más oportunidades en el último mes, de modo que se obtienen diferencias significativas $(p=.0649)$ con respecto a las adolescentes. En cambio, éstas se reconocen más consumidoras de tabaco $(p=.0627) y$, asimismo, informan de que la sustancia que consumen junto con alcohol es otra droga institucionalizada como el tabaco resultando significativas $(p=.0089)$ las diferencias con respecto a los adolescentes varones.

\section{DISCUSIÓN}

Multitud de factores coadyuvan en la conformación y mantenimiento de un fenómeno como el del consumo juvenil de sustancias psicoactivas, definido por una gran raigambre sociocultural e indicadores individuales y psicosociales junto a la acción de conminaciones grupales y contextuales. Investigar sobre los motivadores de consumo de alcohol priorizados e indagar sobre los efectos derivados de cualquier conducta es un paso inexcusable para desarrollar posibles vías de intervención. En esta oportunidad, en líneas generales, los resultados hallados en esta investigación sobre las motivaciones que impulsan al consumo de alcohol en adolescentes van en consonancia con los obtenidos en otros estudios (véase Arévalo, Masip y Abecia, 1997; Alonso, Rosado, Ruiz y Alonso, 1997; Casado, González, Martínez y López, 1998; Espada et al., 2003; Gil y Pons, 2002; Laespada, 2000, 2003; Moral, 2002). En este estudio se ha constatado una motivación para el consumo netamente psicosocial, como es la vinculación y presión grupal, que condiciona la experimentación cuyo trasfondo no es otro que la necesidad de autoafirmación en el grupo asociada a redefiniciones de la identidad. Hecha esta implicación, se aboga por una interpretación psicosocial de la vinculación al grupo de iguales por motivaciones identitarias (Moral, 2002, 2004; Moral et al., 2002; Ovejero, 2000).

A partir del análisis de los factores de protección y riesgo, así como de motivadores de consumo en general, ha de concluirse que se ha confirmado la importancia de la supervisión paternal, de modo que se corrobora que los adolescentes evitan beber porque puede provocar problemas en la vida familiar. A este respecto, baste recordar la acción de los estilos disciplinares paternos y el factor de protección derivado del afecto y apego familiar (Martínez y Robles, 2001; Muñoz-Rivas y Graña, 2001) o la supervisión parental (DiClemente, Wingood, Crosby, Sionean, Cobb, Harrington et al., 2001; Martínez et al., 2003). Asimismo, los adolescentes encuestados son conscientes de los daños inherentes a la instauración de procesos de dependencia (D.G.P.N.S.D., 2003), cuestión constatada en investigaciones en las que se confirma la reducción de la edad de inicio de la dependencia alcohólica (Monrás, 2001) asociada a modificaciones en los patrones de ingesta (Espada, Méndez e Hidalgo, 2000) y cambios en el perfil del enfermo alcohólico (Casa, O’Ferral y Vaca, 2001).

En esta investigación se ha confirmado la existencia de diferencias inter-género en cuestiones relacionadas con los motivadores para el consumo, de manera que las adolescentes reconocen que consumen como mecanismo de evitación del displacer. Ello podría asociarse al distrés emocional (respuesta fisiológica y conductual provocada por el desbordamiento/inadap- 
tación de los mecanismos de afrontamiento del individuo ante una demanda del entorno) (Hervé, 1999). A modo de defensa específica, al recurrir al consumo de drogas el adolescente interpreta que se "palia" la falta o empleo desadaptativo de los recursos individuales, aun cuando lo cierto es que se puede avivar la dependencia del exterior como mecanismo de afrontamiento/huida. En cambio, tal y como se ha explicitado, no se han hallado diferencias en función del género en los motivadores relativos a esparcimiento y prácticas sobreactivadas de ocio, ni en los relativos a la experimentación de nuevas sensaciones, al efecto de modelado grupal o de euforización y desinhibición (véase Martínez-Lorca y Alonso-Sanz, 2003). Por lo que respecta a los hábitos de consumo, se ha confirmado el resultado obtenido en investigaciones precedentes del equipo investigador (véase Moral, 2002; Moral y Ovejero, 2004; Moral et al., 2003; Moral, Ovejero, Rodríguez y Sirvent, 2004) relativo a la constatación de hábitos similares de consumo de alcohol y promedio de intoxicaciones etílicas en chicos y chicas. Asimismo, se ha hallado mayor consumo de tabaco entre las adolescentes, resultado con el que se corrobora la tendencia de consumo avanzada en la revisión teórica (véase Laespada, 2000, 2003; Laespada y Salazar, 1999; Moral, 2002; Suelves y Sánchez-Turet, 2001).

Finalmente, una vez que se ha reflexionado sobre una problemática como la del consumo juvenil de alcohol y otras sustancias psicoactivas, ha de abogarse por complementar la cuantificación de la misma con la implementación de medidas preventivas y/o rehabilitadoras incardinadas en proyectos multidisciplinares. Hemos de comprometernos en estrategias de acción mediante las cuales se tienda al ejercicio de la prevención e intervención psicosocial y comunitaria en materia de drogadicción juvenil basada en el poder del compromiso y la corresponsabilización, en la implementación de medidas que respondan a las bases explicativas y, entre otras muchas labores, en la puesta en práctica de una acción socioeducativa y comunitaria mediante la que se potencien estados de salud personal y social y se intervenga sobre desajustes socioafectivos y crisis de toda índole. Ciertamente, la responsabilización de los contextos individuales en la existencia y resolución de problemas coparticipados está siendo reemplazada por una ampliación del espectro de acción a otras agencias (escuela, padres, comunidad, asociaciones) en propuestas preventivas (Becoña, 2003; Comas, 2001; Garrudo, 2003; Martín y Moncada, 2003; Moral, 2002; Vega, 2000, 2002, 2003). En consecuencia, se propone abogar por la (re)instalación de la dimensión socioeducativa y comunitaria en cuantas parcelas se involucre el adolescente, sin reificar su diversidad y mediante el reconocimiento de su capacidad interpretativa de los escenarios psicosociales e investigando comprehensivamente sobre las bases explicativas coadyuvantes.

\section{REFERENCIAS}

Alonso, J., Rosado, J., Ruiz, R. y Alonso, J. (1997). Consumo de alcohol y adolescencia: Estudio epidemiológico descriptivo. Atención primaria, 19(4), 183-187.

Ambrosio, E. (2003). Vulnerabilidad a la drogadicción. Adicciones, 15(3), 187-189.

Arévalo, J.M., Masip, G.P. y Abecia, L.C. (1997). Consumo de alcohol en una muestra de estudiantes universitarios. Revista Española de Drogodependencias, 22(1), 15-34.

Bach i Bach, L. (2000). La falta de conciencia del daño alcohólico, firme impedimento para la prevención primaria y secundaria del alcoholismo. Revista Española de Drogodependencias, 25(2), 114-117.

Barrio G., Bravo, M.J. y De la Fuente, L. (2000). Consumo de drogas ilegales en España: hacia una diversificación de los patrones de consumo y los problemas asociados. Enf. Emerg., 2, 2-16.

Becoña, E. (2003). Bases científicas de los programas de prevención de las drogodependencias. Madrid: Plan Nacional sobre Drogas.

Bellis, M.A. y Hughes, K. (2003). Consumo recreativo de drogas y reducción de daños en la vida nocturna global. Adicciones, 15 (Supl. 2), 289-309.

Berenzon, S., Medina-Mora, M.E., Carreño, S., Juárez, F., Rojas, E. y Villatoro, J. (1996). Los factores relacionados con el uso y el abuso de sustancias psicoactivas en estudiantes de enseñanza media y media superior de la república mexicana. Salud mental, 19.

Bobes, J., Bascarán, M.T., González, M.P. y Sáiz, P.A. (2000). Epidemiología del uso/abuso del cannabis. Adicciones, 12 (Supl. 2), 31-40.

Bríñez-Horta, J.A. (2001). Diferencias de género en problemas con el alcohol, según el nivel de consumo. Adicciones, 13(4), 439-455.

Calafat, A. y Juan, M. (2003). De la etiología a la prevención del uso y abuso de drogas recreativas. Adicciones, 15 (Supl. 2), 261-287.

Calafat, A., Juan, M., Becoña, E., Fernández, C., Gil, E., Palmer, A., et al. (2000). Salir de marcha y consumo de drogas. Madrid: Plan Nacional sobre Drogas.

Calafat, A., Juan, M., Becoña, E., Fernández, C., Gil, E. y Llopis, J.J. (2000). Consumo y consumidores de cannabis en la vida recreativa. Adicciones, 12 (Supl. 2), 197-230.

Carrasco, A.M. (2000). El consumo de alcohol en los adolescentes españoles en el contexto de sus estilos de vida. Tesis Doctoral inédita. Universidad de Sevilla.

Casa, M.J., O'Ferrall, C. y Vaca, F.J. (2001). Evolución del perfil del enfermo alcohólico durante los últimos veinte 
años. Revista Española de Drogodependencias, 26(2), 127-134.

Casado, C., González, S., Martínez, N. y López, I.L. (1998). Consumo de tabaco, alcohol y cannabis en estudiantes de secundaria de Oviedo. Enfermería científica, 194$195,10-14$

Castellana, M. y Lladó, M. (1999). Adolescencia y juventud: Prevención y percepción del riesgo al consumo. Revista Española de Drogodependencias, 24, 118-130.

Comas, D. (2001). La evaluación de los programas de ocio alternativo de fin de semana. Madrid: Instituto de la Juventud. Ministerio de Trabajo y Asuntos Sociales.

D.G.P.N.S.D. (2000). Encuesta sobre drogas en población escolar, 1998. Informe $n^{\circ} 3$ Marzo 2000. Observatorio Español sobre Drogas. Madrid: DGPNSD. Ministerio del Interior.

D.G.P.N.S.D. (2001). Informe 4. Observatorio Español sobre Drogas. Marzo 2001. Madrid: Ministerio del Interior. Madrid: DGPNSD. Ministerio del Interior.

D.G.P.N.S.D. (2002). Informe 5. Observatorio Español sobre Drogas. Julio 2002. Madrid: Ministerio del Interior.

D.G.P.N.S.D. (2003). Indicadores Tratamiento, Urgencias y Mortalidad. Informe año 2001. Madrid: Ministerio del Interior.

De Crescenzo, L. (2002). Las mujeres son diferentes. El universo femenino de la antigüedad contado por un hombre de hoy. Barcelona: Grijalbo.

DiClemente, R.J., Wingood, G.M., Crosby, R., Sionean, C., Cobb, B.K., Harrington, K., et al. (2001). Parental monitoring: association with adolescents' risk behaviours. Pediatrics, 107, 1363-1368.

Elzo, J., Comas, D., Laespada, M.T., Salazar, L. y Vielva, J. (2000). Las culturas de las drogas en los jóvenes: ritos y fiestas. Servicio de Publicaciones del Gobierno Vasco. Vitoria-Gasteiz.

Espada, J.P., Méndez, F.X. e Hidalgo, M.D. (2000). Consumo de alcohol en escolares: descenso de la edad de inicio y cambios en los patrones de ingesta. Adicciones, 12, 57-64.

Espada, J.P., Méndez, X., Griffin, K. y Botvin, G.J. (2003). Adolescencia: consumo de alcohol y otras drogas. Papeles del Psicólogo, 84, 9-17.

Fernández, C. (2003). Características de los consumidores de drogas recreativas en España y otros países europeos. Adicciones, 15 (Supl. 2), 233-259.

Fernández, J., Alvarez, E., Secades, R., Jiménez, J.M., Cañada, A., Donate, I., et al. (2003). Consumo de drogas de síntesis en estudiantes de secundaria del principado de Asturias (España). Adicciones, 15(1), 31-37.

Fernández-Espejo, E. (2002). Bases neurológicas de la drogadicción. Revista de Neurología, 34(7), 659-664.
Freixa, F. (2000). Percepción crítica del movimiento asociativo en alcoholismo (1950-1999). Revista Española de Drogodependencias, 25(2), 122-157.

Fisher, H. (2000). El primer sexo. Las capacidades innatas de las mujeres y cómo están cambiando el mundo. Madrid: Taurus.

Funes, J. (2000). Drogas y adolescencia. En IGIA. Contextos, sujetos y drogas: un manual sobre drogodependencias. Ed. Pa d'Acció sobre Drogues de Barcelona. Institut Municipal de Salut Pública y FAD. Barcelona.

Gamella, J.F. y Alvarez Roldán, A. (1999). Las rutas del éxtasis. Drogas de síntesis y nuevas culturas juveniles. Barcelona: Ariel.

García, J.M.A. y Carrasco, A.M. (2002). Consumo de alcohol y factores relacionados con el tiempo libre de los jóvenes. Revista de Psicología Social Aplicada, 12(1), 61-78.

García-Portilla, M.P., Sáiz, P., Paredes, B., Martínez, S. y Bobes, J. (2003). Personalidad y uso-abuso de éxtasis (MDMA). Adicciones, 15 (Supl. 2), 199-205.

Garrudo, H. (2003). La cooperación entre la familia, la escuela y las instituciones en un objetivo común: la educación para la salud de niños/as y jóvenes. Revista Española de Drogodependencias, 28(1-2), 104-112.

Gil, M. y Pons, J. (2002). Consumo alcohólico y hábitos de cuidado de la salud en jóvenes de una comunidad urbana periférica. Revista Española de Drogodependencias, 27(1), 22-37.

Graña, J.L. y Muñoz, M.J. (2000). Factores psicológicos de riesgo y de protección para el consumo de drogas en adolescentes. Psicología Conductual, 8, 249-269.

Hervé, F. (1999). Sufrimiento psíquico y toxicomanías. Adicciones, 11(1), 53-63.

Infante, C., Barro, G. y Martín, E. (2003), Tendencias características y problemas asociados al consumo de drogas recreativas en España. Adicciones, 15 (Supl. 2), 77-96.

Juliano, M.D. (2001). Perspectiva de la antropología para estudiar construcciones de género. En: A. Fernández (Coord.). Las mujeres en la enseñanza de las ciencias sociales (pp. 35-59). Madrid: Síntesis.

Laespada, T. (2000). Tabaco y Alcohol. En: J. Elzo (Dir.). La cultura de las drogas en los jóvenes: ritos y fiestas. Bilbao: Universidad de Deusto.

Laespada, M.T. (2003). Consumo de drogas entre escolares donostiarras: un estudio longitudinal durante 21 años. Revista Española de Drogodependencias, 28(1-2), 2447.

Laespada, M.T. y Salazar, L. (1999). Las actividades no formalizadas de los jóvenes. En: J. Elzo, F.A. Orizo, J. González-Anleo, P. González Blasco, M.T. Laespada y L. 
Salazar. Jóvenes españoles'99 (pp. 355-400). Madrid: Fundación Santa María.

León Rubio, J.M. (2000). El modelo biopsicosocial o la concepción social de las acciones de salud. Una guía para la elaboración de la Psicología Social de la Salud. En: A. Ovejero (Ed.). La psicología social en España al filo del año 2000: balance y perspectivas (pp. 274-299). Madrid: Biblioteca Nueva.

Macià, D. (1986). Cuestionario de Actitudes acerca del alcohol y Cuestionario de Información. Método Conductual de Prevención de Drogodependencias. Valencia: Promolibro.

Martín, E. y Moncada, S. (2003). Programas de prevención de ocio alternativo. Adicciones, 15 (Supl. 2), 327-246.

Martínez, J.M. y Robles, L. (2001). Variables de protección ante el consumo de alcohol y tabaco en adolescentes. Psicothema, 13, 222-228.

Martínez, J.L., Fuertes, A., Ramos, M. y Hernández, A. (2003). Consumo de drogas en la adolescencia: importancia del afecto y la supervisión parental. Psicothema, 15(2), 161-166.

Martínez-Lorca, M. y Alonso-Sanz, C. (2003). Búsqueda de sensaciones, autoconcepto, asertividad y consumo de drogas ¿existe relación?. Adicciones, 15(2), 145-157.

Monrás, M. (2001). Cambios en la edad de inicio del tratamiento de la dependencia alcohólica. ¿Debemos cambiar la oferta asistencial?. Adicciones, 13(2), 139-146.

Moral, M.V. (2002). Jóvenes, consumo de sustancia psicoactivas e identidad. Una propuesta de prevención e intervención psicosocial y comunitaria. Tesis Doctoral no publicada. Oviedo: Universidad de Oviedo.

Moral, M.V. y Ovejero, A. (2004). Actitudes ante el consumo de sustancias psicoactivas y mentalidades del usuario en adolescentes de Secundaria. Entemu. UNED Asturias.

Moral, M.V., Ovejero, A. y Rodríguez, F.J. (2002). La construcción intersubejtiva de las diferencias intergénero en el consumo juvenil de alcohol. En: // Symposium Nacional "Tratamiento de la Adicción en la mujer". Madrid.

Moral, M.V., Rodríguez, F.J. y Sirvent, C. (2004). Jóvenes y consumo zocal recreativo de alcohol tras la ley del botellón: politización de las propuestas preventivas. I Congreso de Psicología Jurídica. 12-14 de Julio. Santiago de Compostela.

Moral, M.V., Ovejero, A., Rodríguez, F.J. y Sirvent, C. (2004). Hábitos de consumo de alcohol y otras sustancias psi- coactivas asociados a pautas de ocio juvenil. Revista de Psicología Social Aplicada (en prensa).

Morris, D. (2000). Masculino y femenino. Claves de la sexualidad. Barcelona: Plaza \& Janés.

Muñoz-Rivas, M.J. y Graña, J.L. (2001). Factores familiares de riesgo y de protección para el consumo de drogas en adolescentes. Psicothema, 13, 87-94.

Ovejero, A. (2000). La adicción como búsqueda de identidad: una base teórica psicosocial para una intervención eficaz. Intervención Psicosocial, 9(2), 35-48.

Pascual, F. (2002). Percepción del alcohol entre los jóvenes. Adicciones, 14, suplemento 1, 123-132.

Rooney, J.F. (1990). Funciones sociales de los bares para la juventud en España. Adicciones, 2(3), 209-220.

Sáiz, P.A., González, M.P., Paredes, B., Delgado, J.M., López, J.L., Martínez, S. et al. (2001). Consumo de MMDA (éxtasis) en estudiantes de secundaria. Adicciones, 13(2), 159-171.

Sáiz, P.A., García-Portilla, M.P., Paredes, B. y Bobes, J. (2003). Anfetaminas y drogas de síntesis. En: J. Bobes, M. Casas y M. Gutiérrez (Eds.). Manual de evaluación y tratamiento de drogodependencias. Barcelona: Psiquiatría Editores.

Sánchez, L. (2002). Consumo de alcohol en la población juvenil. Adicciones, 14 (Supl. 1), 99-114.

Sánchez-Carbonell, X. (2004). Contexto cultural y consecuencias legales del consumo de drogas. Trastornos Adictivos, 6(1), 1-4.

Suelves, J.M. y Sánchez-Turet, M. (2001). Asertividad y uso de sustancias en la adolescencia: Resultados de un estudio transversal. Anales de Psicología, 17(1), 15-22.

Vega, A. (2000). El País Vasco ante las drogas. Los retos del proyecto comunitario en las escuelas. San Sebastián. Gakoa.

Vega, A. y otros (2002). Drogas: ¿Qué política para qué prevención?. San Sebastián: Gakoa.

Vega, A. (2003). Repensar la educación sobre las drogas para una nueva prevención. Revista Española de Drogodependencias, 28(1-2), 7-23.

Vega, M.T. y Garrido, E. (2000). Valoración de una intervención preventiva del consumo adolescente de tabaco y alcohol: incidencia de factores personales y situacionales. Revista de Psicología Social y Aplicada, 10(1), 530. 

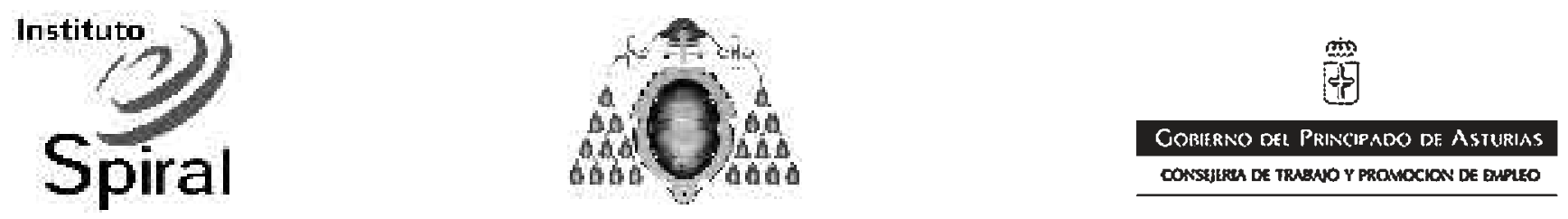

APELLIDOS Y NOMBRE:

EDAD

SEXO $\mathrm{H} M$ (Marcar)

FECHA

A continuación encontrarás una serie de preguntas que debes leer atentamente. Contéstalas de una en una hasta completar el cuestionario. NO pases la hoja hasta terminar de responder a todas las preguntas.

Si tienes alguna duda o pregunta, por favor levanta la mano para que el responsable te la resuelva. ASEGÚRATE DE QUE RESPONDES A TODAS LAS PREGUNTAS y hazlo de la manera más sincera posible. No tienes que poner el nombre, con eso garantizamos tu anonimato. Nadie absolutamente se enterará de tus respuestas, contesta con sinceridad.

Respecto a las preguntas que se presentan a continuación, encontrarás 3 tipos de respuesta: a) aquellas en las cuales debas valorar mediante un número rellenando las casillas; b) aquellas en las que deberás señalar tu opinión con una cruz y c) aquellas que requieren una respuesta abierta. EJEMPLO:

PARA CUMPLIMENTAR EL CUESTIONARIO SIGUE EL SIGUIENTE

$$
\begin{aligned}
& M D=\text { Muy en desacuerdo } \\
& D=\text { Desacuerdo } \\
& I=\text { Inseguro } \\
& A=\text { De acuerdo } \\
& \text { MA = Muy de acuerdo }
\end{aligned}
$$

$$
\begin{aligned}
& 1=\text { Nunca } \\
& 2=\text { Pocas veces (menos } 2) \\
& 3=\text { Bastante }(3 \text { ó } 4 \text { veces) } \\
& 4=\text { Muchas veces (más de } 4) \\
& 5=\text { Casi siempre }
\end{aligned}
$$

Por ejemplo, si un compañero tuyo respondiera ante la afirmación Admiro a quien no consume alcohol, contestar MA significaría que efectivamente está muy de acuerdo con el contenido de la misma. Y que, por tanto, admiraría mucho a una persona que no consumiese alcohol. En cambio, si contestara $\mathbf{D}$ significaría que no admira a la gente que consume alcohol. Por otro lado, si tuviese que responder a la pregunta ¿Consumes bebidas alcohólicas en la actualidad?, contestar 1 significaría que nunca ha consumido bebidas alcohólicas. En cambio, si contestase 4 significaría que tiene un consumo prácticamente diario de bebidas alcohólicas. 


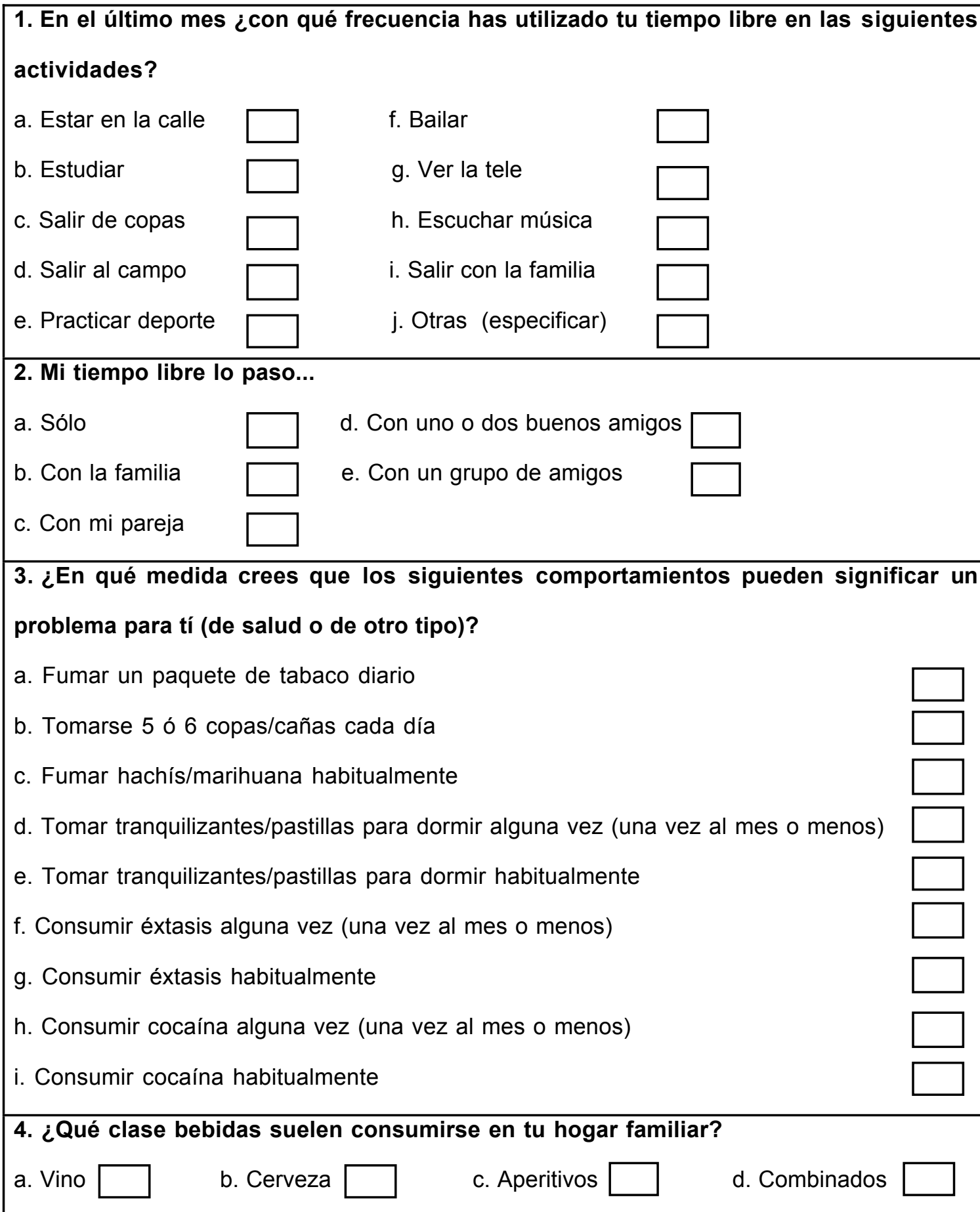


Escribe en cada casilla el número que corresponda:

1 = Nunca; 2 = Pocas veces (menos de 2); 3 = Bastante ( 3 ó 4 veces);

4= Muchas veces (más de 4); 5 = Casi siempre

\begin{tabular}{|c|c|c|}
\hline \multicolumn{3}{|c|}{ 5. ¿Dónde has consumido alcohol en los últimos 30 días? } \\
\hline \multicolumn{3}{|l|}{ a. En casa, yo sólo } \\
\hline \multicolumn{3}{|l|}{ b. Fuera de casa, yo sólo } \\
\hline \multicolumn{3}{|l|}{ c. En casa, con mi familia } \\
\hline \multicolumn{3}{|c|}{ d. En mi casa o en la de un amigo/a, con mis amistades } \\
\hline \multicolumn{3}{|c|}{ e. En bares especialmente baratos con amigos u otra gente } \\
\hline \multicolumn{3}{|c|}{ f. En bares o pubs (normales) con amigos u otra gente } \\
\hline \multicolumn{3}{|c|}{ g. En la calle con amigos u otra gente } \\
\hline \multicolumn{3}{|l|}{ h. En discotecas } \\
\hline \multicolumn{3}{|l|}{ i. En otros sitios } \\
\hline \multicolumn{3}{|c|}{ 6. ¿Por qué bebiste por primera vez? } \\
\hline a. Olvidar & & f. Para ligar \\
\hline b. Evadir la rutina & & g. Para sentir euforia \\
\hline c. Porque bebían mis amigos & & h. Para sentirme más fuerte \\
\hline d. Porque me incitaron a ello & & i. Por curiosidad \\
\hline e. Para desinhibirme & & \\
\hline \multicolumn{3}{|c|}{ 7. ¿En el último mes qué tipo de bebida has tomado y con qué frecuencia? } \\
\hline a. Vino & b. Cerveza & c. Combinados \\
\hline d. Aperitivos-licores $\square$ & e. Kalimocho & f. Otros (Sidra,...) \\
\hline \multicolumn{3}{|c|}{ 8. ¿Porqué crees que bebe la gente? } \\
\hline a. Olvidar & & f. Para ligar \\
\hline b. Evadir la rutina & & g. Para sentir euforia \\
\hline c. Porque beben sus amigos & & h. Para sentirse más fuerte \\
\hline d. Porque les incitaron a ello & & i. Por curiosidad \\
\hline e. Para desinhibirse & & \\
\hline
\end{tabular}


Escribe en cada casilla el número que corresponda:

9. A lo largo de tu vida, ¿cuántas veces has tenido cada uno de los siguientes problemas como consecuencia del consumo de bebidas alcohólicas?

a. Accidente (caída, quemadura, acc. tráfico...) por el que recibiste atención médica

b. Otro problema que necesitó atención médica

c. Detención por la policía o las fuerzas de orden público

d. Faltar al colegio un día entero o más tiempo

e. Riña, discusión u otro conflicto importante sin agresión

f. Pelea o agresión física

10. ¿Porqué vía o vías has recibido, principalmente, información sobre drogas?

a. Padres, hermanos

b. Otros familiares

c. Amigos

d. Profesores

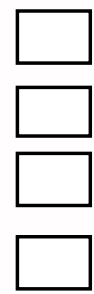

e. Medios de comunicación (TV, prensa, radio)

f. Charlas o cursos sobre el tema

g. Folletos, libros

h. Personas relacionadas con el tema

i. Otros

Dí cual

Señala, mediante una cruz, tu opinión sobre cada pregunta:

$M D=$ Muy en desacuerdo; $D=$ Desacuerdo; I= Inseguro;

$A=$ De acuerdo; $M A=$ Muy de acuerdo

\begin{tabular}{|l|c|c|c|c|c|}
\hline 11. El alcohol en pequeñas cantidades es perjudicial & MD & D & I & A & MA \\
\hline 12. Admiro a quién no consume alcohol o al que deja de beber & MD & D & I & A & MA \\
\hline $\begin{array}{l}\text { 13. Estaría dispuesto a tomar alcohol cuantas veces fuera } \\
\text { necesario hasta "coger el punto" o hasta emborracharme }\end{array}$ & MD & D & I & A & MA \\
\hline $\begin{array}{l}\text { 14. El alcohol puede producir trastornos físicos y mentales } \\
\text { 15. No existe relación entre fumar y tomar alcohol y el consumo de } \\
\text { otras drogas ilegales }\end{array}$ & MD & D & I & A & MA \\
\hline $\begin{array}{l}\text { 16. Estaría dispuesto a comprar una o varias botellas de alcohol } \\
\text { para mi uso (y el de mis amigos) }\end{array}$ & MD & D & I & A & MA \\
\hline
\end{tabular}




\begin{tabular}{|l|c|c|c|c|c|}
\hline 17. No tengo curiosidad para probar nuevas bebidas alcohólicas & MD & D & I & A & MA \\
\hline 18. El abuso de alcohol produce infelicidad a largo plazo & MD & D & I & A & MA \\
\hline $\begin{array}{l}\text { 19. Que tanta gente consuma alcohol demuestra que no es tan } \\
\text { perjudicial como se dice }\end{array}$ & MD & D & I & A & MA \\
\hline 20. Aunque mis amigos beban yo no pienso hacerlo & MD & D & I & A & MA \\
\hline 21. El alcoholismo no tiene cura & MD & D & I & A & MA \\
\hline $\begin{array}{l}\text { 22. Estaría dispuesto a tratar de convencer a alguien sobre el } \\
\text { riesgo del alcohol }\end{array}$ & MD & D & I & A & MA \\
\hline $\begin{array}{l}\text { 23. El alcohol no es uno de los grandes problemas de nuestra } \\
\text { sociedad }\end{array}$ & MD & D & I & A & MA \\
\hline
\end{tabular}

24. Expresa tu grado de acuerdo en las siguientes razones por las que bebes:
a. Olvidar
b. Evadir la rutina
c. Porque beben mis amigos
d. Para desinhibirme
e. Para ligar
f. Para sentir euforia
g. Para sentirme más fuerte

\begin{tabular}{|c|c|c|c|c|}
\hline$M D$ & $D$ & I & $A$ & $M A$ \\
\hline$M D$ & $D$ & I & $A$ & $M A$ \\
\hline$M D$ & $D$ & I & $A$ & $M A$ \\
\hline$M D$ & $D$ & I & $A$ & $M A$ \\
\hline$M D$ & $D$ & I & $A$ & $M A$ \\
\hline$M D$ & $D$ & I & $A$ & $M A$ \\
\hline$M D$ & $D$ & I & $A$ & $M A$ \\
\hline$M D$ & $D$ & I & $A$ & $M A$ \\
\hline$M D$ & $D$ & I & $A$ & $M A$ \\
\hline$M D$ & $D$ & I & $A$ & $M A$ \\
\hline$M D$ & $D$ & I & $A$ & $M A$ \\
\hline$M D$ & $D$ & I & $A$ & $M A$ \\
\hline$M D$ & $D$ & I & $A$ & $M A$ \\
\hline$M D$ & $D$ & I & $A$ & $M A$ \\
\hline$M D$ & $D$ & I & $A$ & $M A$ \\
\hline$M$ & I & $A$ & $M A$ \\
\hline$M$ & I & $A$ & $M A$ \\
\hline$M$
\end{tabular}


Señala, mediante una cruz, tu opinión sobre cada pregunta:

$M D=$ Muy en desacuerdo;

$D=$ Desacuerdo;

I= Inseguro;

$A=$ De acuerdo;

MA= Muy de acuerdo

35. Expresa tu grado de acuerdo en relación con las siguientes razones para no beber:

a. Porque es malo para la salud

b. Porque es demasiado caro

c. Porque es difícil dejarlo una vez que se ha empezado

d. Porque a mis padres les molesta mucho que beba

e. Tomar alcohol engorda

f. Porque no me gusta su sabor

g. Porque sus efectos son molestos (resaca, vómitos, mareos...)

h. Porque beber alcohol provoca actos delictivos

i. Porque va contra mis creencias religiosas

j. Porque provoca accidentes graves

k. Porque provoca problemas en la vida familiar

36. Me siento suficientemente informado en relación con el consumo y los efectos de las drogas

\begin{tabular}{|l|l|l|l|l|}
\hline MD & D & I & A & MA \\
\hline MD & D & I & A & MA \\
\hline MD & D & I & A & MA \\
\hline MD & D & I & A & MA \\
\hline MD & D & I & A & MA \\
\hline MD & D & I & A & MA \\
\hline MD & D & I & A & MA \\
\hline MD & D & I & A & MA \\
\hline MD & D & I & A & MA \\
\hline MD & D & I & A & MA \\
\hline MD & D & I & A & MA \\
\hline MD & D & I & A & MA \\
\hline
\end{tabular}

37. En los últimos 30 días ¿Cuántos días has salido con tus amigos para divertirte? (poner cantidad de días por semana)

38. En caso de que hayas salido de noche en los últimos 30 días ¿a qué hora volviste a casa la última vez que saliste en fin de semana? (especificar franja horaria)

39. ¿Consumes bebidas alcohólicas en la actualidad?

40. ¿A qué edad empezaste a consumir alcohol por primera vez? (años)

41. En el último mes ¿te has emborrachado alguna vez? (rodear) $\mathrm{SI} \quad \mathrm{NO}$

Número de veces

42. ¿Fumas? (rodear) SI NO

Número de cigarrillos en 24 horas

43. ¿Con qué frecuencia has consumido bebidas alcohólicas en los últimos 30 días?

a. Días laborales

$\mathrm{N}^{\circ}$ de días

b. Fines de semana

$\mathrm{N}^{\circ}$ de fines de semana

44. ¿Cuánto dirías que bebes?

En exceso

Con moderación

Poco 